\title{
On the Role of Nonsynchronous Rotating Damping in Rotordynamics
}

\author{
GIANCARLO GENTA* and EUGENIO BRUSA \\ Dipartimento di Meccanica, Politecnico di Torino, C.so Duca degli Abruzzi, 24-I 10129 Torino, Italy \\ (Received 29 January 1999; Revised 15 May 1999; In final form 31 May 1999)
}

\begin{abstract}
Nonsynchronous rotating damping, i.e. energy dissipations occurring in elements rotating at a speed different from the spin speed of a rotor, can have substantial effects on the dynamic behaviour and above all on the stability of rotating systems.

The free whirling and unbalance response for systems with nonsynchronous damping are studied using Jeffcott rotor model. The system parameters affecting stability are identified and the threshold of instability is computed. A general model for a multi-degrees of freedom model for a general isotropic machine is then presented. The possibility of synthesizing nonsynchronous rotating and nonrotating damping using rotor- and stator-fixed active dampers is then discussed for the general case of rotors with many degrees of freedom.
\end{abstract}

Keywords: Mechanical design, Rotordynamics, Structural damping, Active damping

\section{INTRODUCTION}

Damping plays different roles in rotordynamics. It is well known that nonrotating damping is always stabilizing, while the damping of the rotating parts of the machine has a destabilizing effect on whirling motions occurring in supercritical conditions (Dimentberg, 1961; Lalanne and Ferraris, 1990; Genta, 1998). The effect of damping depends on whether the energy dissipation occurs in stationary or rotating parts of the machine: an eddy current damper with a stationary permanent magnet and a rotating conducting disc can cause severe instability in the supercritical regime (Genta et al., 1996).

Apart from dampers which are stationary or rotate at the same spin speed of the rotor, there can be cases in which energy is dissipated in an element rotating at a different speed, both in the same direction of the rotor (co-rotating damping) or in the opposite direction (counter-rotating damping).

A very well known case is the heuristic model of lubricated bearings (Crandall, 1956; 1982; Lund, 1987). The oil film is assumed to rotate at half the spin speed, with the result of giving way to an

\footnotetext{
* Corresponding author. Tel.: 003911 5646922. Fax: 003911 5646999. E-mail: genta@polito.it.
} 
unstable behaviour, usually referred to as oil whip. The threshold of instability is located at a speed identified on the Campbell diagram by the intersection of the curve related to the first forward mode with the line of equation $\lambda=\omega / 2$. In many cases its value is close to twice the first critical speed.

This model has been generalized (Muszynska, 1986; 1990; 1991; 1995; Muszynska and Bently, 1989; Muszynska and Grant, 1991) to include the case of fluid rotating with an average velocity different from $\lambda=\omega / 2$. The fluid is so modelled as a damper rotating at a speed $\omega_{\mathrm{d}}=r \omega$ (in the mentioned papers, the symbol $\lambda$ is used for the ratio $\omega_{\mathrm{d}} / \omega$; here a different symbol has been introduced to avoid confusion with the whirl speed $\lambda$ ), obtaining a deeper insight in the fluid-induced instabilities of rotating machinery. In Muszynska (1989) the antiswirl effect obtained by injecting a fluid in the rotor-stator clearance in a direction opposite to rotation is studied in terms of nonsynchronous rotating damping, eventually counterrotating. This model can be used for fluid-rotor interactions of all types, as those occurring in bearings, seals, blade-tip gaps, etc., and show that the threshold of instability is very much affected by ratio $r$, strongly increasing with decreasing $r$.

The results regarding nonsynchronous rotating damping found in the literature are usually limited to the case of co-rotation of the damping or slow counter-rotation, with speeds lower than the spin speed.

The availability of active dampers has recently broadened the application of nonsynchronous damping to cases in which no fluid-rotor interaction is involved. In rotors supported in active magnetic bearings (AMB), the damping effect is due to the derivative action of the control loop which keeps the rotor in position. Active dampers can also be added to rotors supported in conventional bearings, using techniques derived from AMB technology.

If an active device is present, nonsynchronous damping can be synthesized without the need of resorting to a material device which actually rotates at the required speed: the damper can be stator-fixed or even rotor-fixed and the nonsynchronous damping action can be obtained by choosing a suitable control law. In particular, if digital techniques are used, the choice of the speed ratio $r$ can be defined by the program of the control microprocessor.

In the case of statorless rotors, e.g. spinning spacecrafts, a damping action rotating at a speed different from that of rotation can be easily obtained using active dampers. Stability can thus be achieved even in cases for which passive stabilization is difficult (Genta and Brusa, 1997; Nobili et al., 1998).

The aim of the present paper is studying the effects of nonsynchronous damping on the dynamic behaviour of rotors, firstly using the Jeffcott rotor model and then a general multi-degrees of freedom isotropic model. The control laws which allow one to synthesize such damping action will then be dealt with in detail.

\section{JEFFCOTT ROTOR WITH NONSYNCRONOUS DAMPING}

\section{Equations of Motion}

Consider a damped Jeffcott rotor running at constant speed (Genta, 1998). Assume that a damper, with damping coefficient $c_{\mathrm{d}}$, rotating at a speed $\omega_{\mathrm{d}}=r \omega$, is present together with the usual nonrotating and rotating damping $c_{\mathrm{n}}$ and $c_{\mathrm{r}}$.

By resorting to the complex coordinate $z=x+\mathrm{i} y$ (Dimentberg, 1961; Genta, 1998), the equation of motion can be written in the form

$$
m \ddot{z}+c \dot{z}+\left(k-\mathrm{i} \omega c_{\mathrm{r}}-\mathrm{i} \omega_{\mathrm{d}} c_{\mathrm{d}}\right) z=F_{\mathrm{r}} \mathrm{e}^{\mathrm{i} \omega t}+F_{\mathrm{n}},
$$

where $m, k$ and $c$ are the mass, stiffness and total damping $\left(c=c_{\mathrm{n}}+c_{\mathrm{r}}+c_{\mathrm{d}}\right)$, respectively. Force $F_{\mathrm{r}}$ rotates at the spin speed $\left(F_{\mathrm{r}}=m \varepsilon \omega^{2}\right.$ if it is due to the eccentricity $\varepsilon$ of the centre of mass) and $F_{\mathrm{n}}$ is fixed in the inertial frame, although possibly being time-dependent. A general nonsynchronous force, $F_{\text {ns }} \mathrm{e}^{\mathrm{i} \omega^{*} t}$, rotating at a speed $\omega^{*}$ different from $\omega$, can be added. 
Note that the same equation can be written using real coordinates, obtaining

$$
\begin{gathered}
{\left[\begin{array}{cc}
m & 0 \\
0 & m
\end{array}\right]\left\{\begin{array}{l}
\ddot{x} \\
\ddot{y}
\end{array}\right\}+\left[\begin{array}{ll}
c & 0 \\
0 & c
\end{array}\right]\left\{\begin{array}{l}
\dot{x} \\
\dot{y}
\end{array}\right\}} \\
+\left[\begin{array}{cc}
k & \omega c_{\mathrm{r}}+\omega_{\mathrm{d}} c_{\mathrm{d}} \\
-\omega c_{\mathrm{r}}-\omega_{\mathrm{d}} c_{\mathrm{d}} & k
\end{array}\right]\left\{\begin{array}{l}
x \\
y
\end{array}\right\} \\
=\left\{\begin{array}{c}
F_{\mathrm{r}} \cos (\omega t) \\
F_{\mathrm{r}} \sin (\omega t)
\end{array}\right\}+\left\{\begin{array}{l}
F_{\mathrm{n}_{x}} \\
F_{\mathrm{n}_{y}}
\end{array}\right\} .
\end{gathered}
$$

Equation (2) shows clearly that a circulatory matrix is present due to the rotating (synchronous and nonsynchronous) damping. The damping matrix has a stabilizing effect while the circulatory one reduces the stability of the system.

\section{Free Whirling}

The homogeneous equation associated with Eq. (1) allows one to study the stability of the system. By assuming a solution of the type $z=z_{0} \mathrm{e}^{\mathrm{i} \lambda t}$, the following characteristic equation is obtained:

$$
-m \lambda^{2}+\mathrm{i} c \lambda+k-\mathrm{i} \omega c_{\mathrm{r}}-\mathrm{i} \omega_{\mathrm{d}} c_{\mathrm{d}}=0 .
$$

By introducing the nondimensional whirl and spin speeds $\lambda^{\prime}=\lambda / \omega_{\mathrm{cr}}, \omega^{\prime}=\omega / \omega_{\mathrm{cr}}$ and $\omega_{\mathrm{d}}^{\prime}=\omega_{\mathrm{d}} / \omega_{\mathrm{cr}}$, where $\omega_{\mathrm{cr}}=\sqrt{\mathrm{k} / \mathrm{m}}$ is the critical speed of the undamped system, and the damping ratios $\zeta_{i}$, defined in the usual way (Genta, 1998), the solutions of the characteristic equation can be shown to be

$$
\lambda^{\prime}=\mathrm{i} \zeta \pm(2 \Gamma-2 \mathrm{i} \Xi)^{1 / 2}
$$

where $\Gamma=\left(1-\zeta^{2}\right) / 2$ and $\Xi=\omega^{\prime} \zeta_{\mathrm{r}}+\omega_{\mathrm{d}}^{\prime} \zeta_{\mathrm{d}}$.

The real part of the nondimensional complex whirl speed, i.e. the nondimensional whirl frequency is

$$
\Re\left(\lambda^{\prime}\right)= \pm\left[\Gamma+\left(\Gamma^{2}+\Xi^{2}\right)^{1 / 2}\right]^{1 / 2}
$$

where the $(+)$ and $(-)$ signs indicate forward and backward whirling, respectively.
The expression of the imaginary part of the complex whirl speed, i.e. the decay rate, depends on the sign of the nondimensional parameter $\Xi$ :

$$
\Im\left(\lambda^{\prime}\right)=\left\{\begin{array}{l}
\zeta \mp\left[-\Gamma+\left(\Gamma^{2}+\Xi^{2}\right)^{1 / 2}\right]^{1 / 2} \\
\text { if } \Xi \geq 0, \\
\zeta \pm\left[-\Gamma+\left(\Gamma^{2}+\Xi^{2}\right)^{1 / 2}\right]^{1 / 2} \\
\text { if } \Xi<0,
\end{array}\right.
$$

where the upper and lower signs indicate forward and backward whirling, respectively.

Stability when $\Xi \geq 0$ From Eq. (6) it follows immediately that the backward mode is always stable. The condition for stability of the forward mode is

$$
\zeta>\left[-\Gamma+\left(\Gamma^{2}+\Xi^{2}\right)^{1 / 2}\right]^{1 / 2}
$$

i.e.

$$
\omega^{\prime}<\frac{\zeta}{\zeta_{\mathrm{r}}}-\omega_{\mathrm{d}}^{\prime} \frac{\zeta_{\mathrm{d}}}{\zeta_{\mathrm{r}}}
$$

By introducing the ratio $r=\omega_{\mathrm{d}}^{\prime} / \omega^{\prime}$ between the speed of rotation of the damper $c_{\mathrm{d}}$ and the spin speed, the condition for stability becomes

$$
\omega^{\prime}<\frac{\zeta}{\zeta_{\mathrm{r}}+r \zeta_{\mathrm{d}}} .
$$

A particular case is that of counter-rotating damping with $r=-1$. The nondimensional threshold of instability, which for synchronous damping has a value $\zeta / \zeta_{\mathrm{r}}$, is now equal to $\zeta /\left(\zeta_{\mathrm{r}}-\zeta_{\mathrm{d}}\right)$.

Stability when $\Xi<0$ In this case the forward mode is always stable. The condition for stability of the backward mode is

$$
\zeta>\left[-\Gamma+\left(\Gamma^{2}+\Xi^{2}\right)^{1 / 2}\right]^{1 / 2},
$$

i.e.

$$
\omega^{\prime}<-\frac{\zeta}{\zeta_{\mathrm{r}}}-\omega_{\mathrm{d}}^{\prime} \frac{\zeta_{\mathrm{d}}}{\zeta_{\mathrm{r}}} .
$$


or

$$
\omega^{\prime}<\frac{\zeta}{-\zeta_{\mathrm{r}}-r \zeta_{\mathrm{d}}}
$$

Conclusions on stability A stability chart is shown in Fig. 1. Forward modes are always stable for counter-rotating damping with $\left|\omega_{\mathrm{d}}^{\prime}\right|>\omega^{\prime} \zeta_{\mathrm{r}} / \zeta_{\mathrm{d}}$, while for counter-rotating damping with a speed lower (in absolute value) than the mentioned value and for co-rotating damping the condition for stability is expressed by Eq. (7).

Backward modes are always stable for corotating or for counter-rotating damping with $\left|\omega_{\mathrm{d}}^{\prime}\right|<\omega^{\prime} \zeta_{\mathrm{r}} / \zeta_{\mathrm{d}}$, while for counter-rotating damping with a speed higher (in absolute value) than the mentioned value the condition for stability is expressed by Eq. (9).

It is possible to find a value of $r$ which assures a stable working of the machine at any speed for each value of rotating damping. A fairly large zone about $r \zeta_{\mathrm{d}} / \zeta_{\mathrm{r}}=1$, where the value of the threshold of stability is very high, can be found. No threshold of instability exists if $r \zeta_{\mathrm{d}} / \zeta_{\mathrm{r}}=1$.

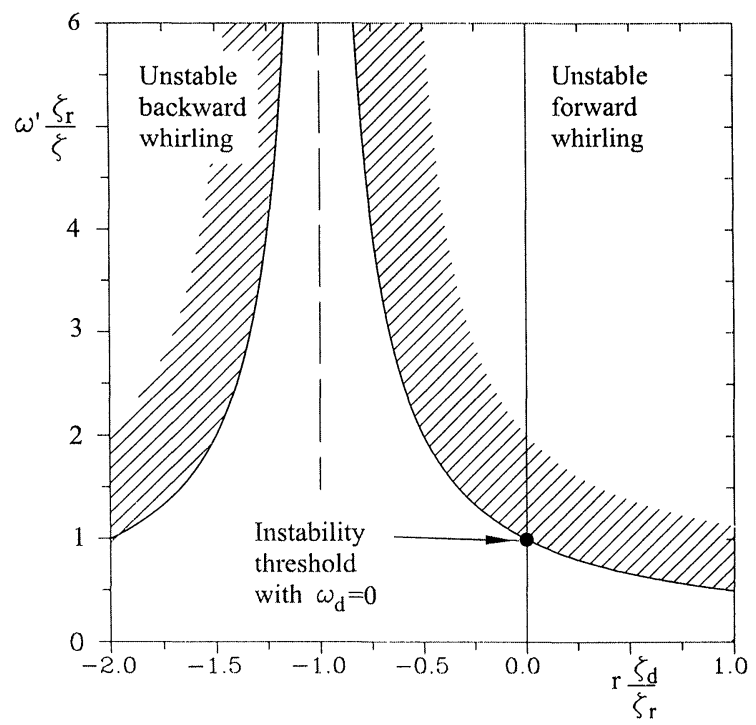

FIGURE 1 Stability chart for the forward and backward whirl modes. Nondimensional parameter $\omega^{\prime} \zeta_{\mathrm{r}} / \zeta$ as a function of $r \zeta_{\mathrm{d}} / \zeta_{\mathrm{r}}$.
Note that nonsynchronous damping can make the system unstable even at standstill (i.e. for $\omega=0$ ), the condition for stability being

$$
\left|\omega_{\mathrm{d}}^{\prime}\right|<\frac{\zeta_{\mathrm{n}}+\zeta_{\mathrm{r}}+\zeta_{\mathrm{d}}}{\zeta_{\mathrm{d}}}
$$

In the case of nonrotating systems the sign of $\omega_{\mathrm{d}}$ has no meaning as the direction of rotation of the damper is immaterial.

\section{Unbalance Response}

The unbalance response can be computed by introducing a solution of the type $z=z_{0} \mathrm{e}^{\mathrm{i} \omega t}$ into Eq. (1). The relevant algebraic equation yielding the amplitude of the response is then

$$
\left[1-\omega^{\prime 2}+2 \mathrm{i} \zeta_{\mathrm{n}} \omega^{\prime}+2 \mathrm{i}\left(\omega^{\prime}-\omega_{\mathrm{d}}^{\prime}\right) \zeta_{\mathrm{d}}\right] z_{0}=\varepsilon \omega^{\prime 2}
$$

The real and imaginary parts and the absolute value of the nondimensional response are

$$
\begin{aligned}
& \Re\left(\frac{z_{0}}{\varepsilon}\right) \\
& =\frac{\omega^{\prime 2}\left(1-\omega^{\prime 2}\right)}{\left(1-\omega^{\prime 2}\right)^{2}+4 \omega^{\prime 2}\left[\zeta_{\mathrm{n}}+\left(1-\omega_{\mathrm{d}}^{\prime} / \omega^{\prime}\right) \zeta_{\mathrm{d}}\right]^{2}} \\
& \Im\left(\frac{z_{0}}{\varepsilon}\right) \\
& =\frac{2 \omega^{\prime 3}\left[\zeta_{\mathrm{n}}+\left(1-\omega_{\mathrm{d}}^{\prime} / \omega^{\prime}\right) \zeta_{\mathrm{d}}\right]}{\frac{z_{0}}{\varepsilon} \mid} \frac{\left(1-\omega^{\prime 2}\right)^{2}+4 \omega^{\prime 2}\left[\zeta_{\mathrm{n}}+\left(1-\omega_{\mathrm{d}}^{\prime} / \omega^{\prime}\right) \zeta_{\mathrm{d}}\right]^{2}}{\left\{\left(1-\omega^{\prime 2}\right)^{2}+4 \omega^{\prime 2}\left[\zeta_{\mathrm{n}}+\left(1-\omega_{\mathrm{d}}^{\prime} / \omega^{\prime}\right) \zeta_{\mathrm{d}}\right]^{2}\right\}^{1 / 2}} .
\end{aligned}
$$

\section{Systems with Structural Damping}

In many cases both rotating and nonrotating damping can best be modelled as hysteretic damping. 
The nonsynchronous damper will be assumed to be of the viscous type and to have a damping coefficient equal to $c_{\mathrm{d}}$. By resorting to the usual small damping assumptions for hysteretic damping (Ramanujam and Bert, 1983; Genta, 1998), the characteristic equation for free whirling can be shown to be

$$
-m \lambda^{2}+k+\mathrm{i}\left[ \pm \eta_{\mathrm{n}} k_{\mathrm{n}} \pm \eta_{\mathrm{r}} k_{\mathrm{r}}+c_{\mathrm{d}}\left(\lambda-\omega_{\mathrm{d}}\right)\right]=0
$$

where the first double sign is $(+)$ for forward whirling and (-) for backward whirling, while the second one is $(+)$ in subcritical conditions and (-) in supercritical conditions and backward whirling. For a detailed discussion of the approximations underlying Eq. (14), see Genta (1998).

The solution of the characteristic equation is

$$
\lambda=\frac{\mathrm{i} c_{\mathrm{d}}}{2 m} \pm(2 \Gamma+2 \mathrm{i} \Xi)^{1 / 2},
$$

where $\quad \Gamma=\left(4 k m-c_{\mathrm{d}}^{2}\right) / 8 m^{2}$ and $\quad \Xi=\left( \pm \eta_{n} k_{n} \pm\right.$ $\left.\eta_{\mathrm{r}} k_{\mathrm{r}}-c_{\mathrm{d}} \omega_{\mathrm{d}}\right) / 2 m$

The real part of the complex whirl speed, i.e. the whirl frequency, is still given by Eq. (5), while its imaginary part, which depends on the sign of the parameter $\Xi$ (in the present case it is not nondimensional), is still given by Eq. (6), in which $c_{\mathrm{d}} /(2 \mathrm{~km})^{1 / 2}$ is substituted for $\zeta$.

The stability assessment is in this case less straightforward, as the expression for $\Xi$ depends whether the rotor is working in subcritical forward whirling, supercritical forward whirling or backward whirling conditions.

In the first case (subcritical forward whirling), the expression for $\Xi$ is

$$
\Xi=\frac{\eta_{\mathrm{n}} k_{\mathrm{n}}+\eta_{\mathrm{r}} k_{\mathrm{r}}-c_{\mathrm{d}} \omega_{\mathrm{d}}}{2 m}
$$

It can he readily shown that if $\Xi>0$ the motion is always stable.

If $\Xi<0$ the condition for stability is

$$
\omega_{\mathrm{d}}<\frac{\eta_{\mathrm{n}} k_{\mathrm{n}}+\eta_{\mathrm{r}} k_{\mathrm{r}}}{c_{\mathrm{d}}}+\omega_{\mathrm{cr}} .
$$

Condition (16) states that speed $\omega_{\mathrm{d}}$ must be either negative (counter-rotating damping) or small enough in case of a co-rotating damper.

In the second case (supercritical forward whirling), the expression for $\Xi$ is

$$
\Xi=\frac{\eta_{\mathrm{n}} k_{\mathrm{n}}-\eta_{\mathrm{r}} k_{\mathrm{r}}-c_{\mathrm{d}} \omega_{\mathrm{d}}}{2 m}
$$

If $\Xi>0$ the motion is always stable.

If $\Xi<0$ the condition for stability is

$$
\omega_{\mathrm{d}}<\frac{\eta_{\mathrm{n}} k_{\mathrm{n}}-\eta_{\mathrm{r}} k_{\mathrm{r}}}{c_{\mathrm{d}}}+\omega_{\mathrm{cr}}
$$

Again condition (17) states a maximum value for $\omega_{\mathrm{d}}$ for achieving stability. Nonsynchronous damping enhances stability when $\omega_{\mathrm{d}}<\omega_{\mathrm{cr}}$ and acts to reduce stability if $\omega_{\mathrm{d}}>\omega_{\mathrm{cr}}$.

In the third case (backward whirling), the expression for $\Xi$ is

$$
\Xi=\frac{-\eta_{\mathrm{n}} k_{\mathrm{n}}-\eta_{\mathrm{r}} k_{\mathrm{r}}-c_{\mathrm{d}} \omega_{\mathrm{d}}}{2 m}
$$

If $\Xi<0$ the motion is always stable if $\omega_{\mathrm{d}}>0$, i.e. if the damper is co-rotating. In case of counterrotating damping the condition for stability is

$$
\left|\omega_{\mathrm{d}}\right|<\frac{\eta_{\mathrm{n}} k_{\mathrm{n}}+\eta_{\mathrm{r}} k_{\mathrm{r}}}{c_{\mathrm{d}}}
$$

If $\Xi>0$ the condition for stability is

$$
\left|\omega_{\mathrm{d}}\right|<\frac{\eta_{\mathrm{n}} k_{\mathrm{n}}+\eta_{\mathrm{r}} k_{\mathrm{r}}}{c_{\mathrm{d}}}+\omega_{\mathrm{cr}}
$$

If $\omega_{\mathrm{d}}$ is positive or negative with $\left|\omega_{\mathrm{d}}\right|<\omega_{\mathrm{cr}}$ nonsynchronous damping is stabilizing, while its effect is opposite and if $\left|\omega_{\mathrm{d}}\right|>\omega_{\mathrm{cr}}$.

\section{ROTOR WITH MANY DEGREES OF FREEDOM}

The general equation of an isotropic, multi-degrees of freedom rotor with nonsynchronous damping can be written, with reference to the complex 
coordinates $\mathbf{q}$, in the form described in Genta (1998):

$$
\begin{gathered}
\mathbf{M} \ddot{\mathbf{q}}+(C-\mathrm{i} \omega \mathbf{G}) \dot{\mathbf{q}}+\left(\mathbf{K}+\mathbf{K}_{\omega} \omega^{2}-\mathrm{i} \omega \mathbf{C}_{\mathrm{r}}-\mathrm{i} \omega_{\mathrm{d}} \mathbf{C}_{\mathrm{d}}\right) \mathbf{q} \\
=\mathbf{F}_{\mathrm{n}}+\omega^{2} \mathbf{F}_{\mathrm{r}} \mathrm{e}^{\mathrm{i} \omega t} .
\end{gathered}
$$

Matrix $\mathbf{K}_{\omega}$ has been introduced to take into account centrifugal stiffening and $\mathbf{C}=\mathbf{C}_{\mathbf{n}}+$ $\mathbf{C}_{\mathrm{r}}+\mathbf{C}_{\mathrm{d}}$. The rotating forcing function has been assumed to be due to unbalance and factor $\omega^{2}$ has consequently been extracted from vector $\mathbf{F}_{\mathrm{r}}$ to state it explicitly.

Equation (20) can be used for the study of free and forced behaviour and the stability of the system. Although little can be said in general, the conclusions drawn from the Jeffcott rotor, i.e. that counter-rotating damping generally increases the stability of forward modes while decreasing that of backward ones, still holds in a qualitative way.

\section{SYNTHESIZING ROTATING AND NONROTATING DAMPING}

\section{Stator-fixed Active Damper}

As already stated, a material device which rotates at the nonsynchronous speed $\omega_{\mathrm{d}}$ is not needed, if active dampers are used. Consider a rotor with many degrees of freedom with both rotating and nonrotating damping, provided of an active damper supplying the control force $\mathbf{F}_{\mathrm{c}}$. Equation (20) modifies as

$$
\begin{aligned}
& \mathbf{M} \ddot{\mathbf{q}}+(\mathbf{C}-\mathrm{i} \omega \mathbf{G}) \dot{\mathbf{q}}+\left(\mathbf{K}+\mathbf{K}_{\omega} \omega^{2}-\mathrm{i} \omega \mathbf{C}_{\mathrm{r}}\right) \mathbf{q} \\
& \quad=\mathbf{F}_{\mathrm{n}}+\omega^{2} \mathbf{F}_{\mathrm{r}} \mathrm{e}^{\mathrm{i} \omega t}+\mathbf{F}_{\mathrm{c}} .
\end{aligned}
$$

To simulate a nonsynchronous damping rotating at the speed $\omega_{\mathrm{d}}$, the control force $\mathbf{F}_{\mathrm{c}}$ must be, in terms of complex coordinates,

$$
\mathbf{F}_{\mathrm{c}}=-\mathbf{C}_{\mathrm{d}} \dot{\mathbf{q}}+\mathrm{i} \omega_{\mathrm{d}} \mathbf{C}_{\mathrm{d}} \mathbf{q}
$$

i.e., splitting it into its components in $x z$ and $y z$ planes:

$$
\left\{\begin{array}{l}
\mathbf{F}_{\mathrm{c}_{x}} \\
\mathbf{F}_{\mathrm{c}_{y}}
\end{array}\right\}=\left\{\begin{array}{l}
-\mathbf{C}_{\mathrm{d}} \Re(\dot{\mathbf{q}})-\omega_{\mathrm{d}} \mathbf{C}_{\mathrm{d}} \Im(\mathbf{q}) \\
-\mathbf{C}_{\mathrm{d}} \Im(\dot{\mathbf{q}})+\omega_{\mathrm{d}} \mathbf{C}_{\mathrm{d}} \Re(\mathbf{q})
\end{array}\right\}
$$

The equation of motion (21) can be written with reference to the state space in the usual way

$$
\dot{\mathbf{z}}=\mathbf{A z}+\mathbf{B}_{\mathrm{n}} \mathbf{u}_{\mathrm{n}}+\mathbf{B}_{\mathrm{r}} \mathbf{u}_{\mathrm{r}} \omega^{2}+\mathbf{B}_{\mathrm{c}} \mathbf{u}_{\mathrm{c}}
$$

where the real state vector is

$$
\mathbf{z}=\left[\Re(\dot{\mathbf{q}})^{\mathrm{T}} \Im(\dot{\mathbf{q}})^{\mathrm{T}} \Re(\mathbf{q})^{\mathrm{T}} \Im(\mathbf{q})^{\mathrm{T}}\right]^{\mathrm{T}}
$$

and the dynamic matrix is

$$
\begin{aligned}
& \mathbf{A}= \\
& {\left[\begin{array}{cccc}
-\mathbf{M}^{-1} \mathbf{C} & -\omega \mathbf{M}^{-1} \mathbf{G} & -\mathbf{M}^{-1}\left(\mathbf{K}+\omega^{2} \mathbf{K}_{\omega}\right) & -\omega \mathbf{M}^{-1} \mathbf{C}_{\mathrm{r}} \\
\omega \mathbf{M}^{-1} \mathbf{G} & -\mathbf{M}^{-1} \mathbf{C} & \omega \mathbf{M}^{-1} \mathbf{C}_{r} & -\mathbf{M}^{-1}\left(\mathbf{K}+\omega^{2} \mathbf{K}_{\omega}\right) \\
\mathbf{I} & \mathbf{0} & \mathbf{0} & \mathbf{0} \\
\mathbf{0} & \mathbf{I} & \mathbf{0} & \mathbf{0}
\end{array}\right]}
\end{aligned}
$$

If the nonrotating and rotating inputs $\mathbf{u}_{\mathrm{n}}$ and $\mathbf{u}_{\mathrm{r}}$ reduce to the corresponding force vectors $\mathbf{F}_{\mathrm{n}}$ and $\mathbf{F}_{\mathrm{r}}$, the input gain matrices are simply

$$
\mathbf{B}_{\mathrm{n}}=\mathbf{B}_{\mathrm{r}}=\left[\begin{array}{c}
\mathbf{M}^{-1} \\
\mathbf{0}
\end{array}\right] \text {. }
$$

The simplest way to synthesize nonsynchronous rotating damping is by using a number of actuators and sensors positioned at given locations of the rotor. In the following it will be assumed that each actuator is able to supply two control forces in directions $x$ and $y$ and that the force due to the $i$ th actuator is assumed as the $i$ th control input

$$
\mathbf{u}_{\mathrm{c}_{i}}=\left[\begin{array}{ll}
\mathbf{F}_{\mathrm{c}_{x_{i}}} & \mathbf{F}_{\mathrm{c}_{y_{i}}}
\end{array}\right]^{\mathrm{T}} \text {. }
$$

The corresponding input gain matrix is then

$$
\mathbf{B}_{\mathrm{c}_{i}}=\left[\begin{array}{c}
\mathbf{M}^{-1} \delta_{i} \\
\mathbf{0}
\end{array}\right],
$$

where $\delta_{i}$ is a matrix with $n$ rows ( $n$ is the number of the real degrees of frecdom of the system) and 2 columns

$$
\delta_{i}=\left[\begin{array}{lllll}
\ldots & 1 & \ldots & 0 & \ldots \\
\ldots & 0 & \ldots & 1 & \ldots
\end{array}\right]^{\mathrm{T}},
$$


with the two nonvanishing entries in the $r$ th and $s$ th columns, corresponding to the degrees of freedom for the displacements at the actuator location in $x$ and $y$ directions respectively.

Each sensor is assumed to read the velocities and the displacements in directions $x$ and $y$ at a given location, which in general is not the same as the corresponding actuator location (non-co-located actuators and sensors).

The $i$ th output vector is then

$$
\mathbf{y}_{i}=\left[\begin{array}{llll}
v_{x_{i}} & y_{u_{i}} & x_{i} & y_{i}
\end{array}\right]^{\mathrm{T}} .
$$

The output-gain matrix is then a matrix with 4 rows and $2 n$ columns having the structure

$$
\mathbf{C}_{i}=\left[\begin{array}{cc}
\delta_{i}^{\prime \mathrm{T}} & \mathbf{0} \\
\mathbf{0} & \delta_{i}^{\prime \mathrm{T}}
\end{array}\right],
$$

where matrix $\delta_{i}^{\prime}$ is the same as matrix $\delta_{i}$ with the only difference that the nonzero elements are located at the $r^{\prime}$ th and $s^{\prime}$ th rows corresponding to the sensor positions. The two matrices are exactly the same in the case of co-located sensors and actuators.

In the simple case of decentralized control, in which the $i$ th actuator is driven by the $i$ th sensor, an ideal proportional control law can be stated by simply closing the loop with the constant-gain matrix $\mathbf{P}_{i}$

$$
\mathbf{u}_{\mathrm{c}_{i}}=\mathbf{P}_{i} \mathbf{y}_{i},
$$

where

$$
\mathbf{P}=-c_{\mathrm{d}_{i}}\left[\begin{array}{cccc}
1 & 0 & 0 & -\omega_{\mathrm{d}} \\
0 & 1 & \omega_{\mathrm{d}} & 0
\end{array}\right] .
$$

This control-gain matrix allows one to synthesize exactly a nonsynchronous damper with constant damping coeffcient $c_{\mathrm{d}_{i}}$ rotating at the speed $\omega_{\mathrm{d}}$. It is clearly a highly idealized result, as no sensor, actuator, or controller dynamics has been taken into account and the velocity at the sensor location has been assumed to be readily available.

In any practical application, the actual transfer function of the various elements in the control loop must be carefully accounted for and a more complex control law, at least of the PID type, must be implemented. The standard technology developed for active magnetic bearings can however be used.

\section{Rotor-fixed Active Damper}

The active damper considered in the previous section is stator-fixed, i.e. is physically not rotating. The same effect can however be obtained by using an active damper which rotates together with the rotor, as it is mandatory, for instance, in the case of statorless (or free in Crandall, 1995) rotors. In such a case the equation of motion is better written in a rotor-fixed reference frame, obtaining

$$
\begin{gathered}
\mathbf{M} \ddot{\mathbf{r}}+[\mathbf{C}-\mathrm{i} \omega(2 \mathbf{M}-\mathbf{G})] \dot{\mathbf{r}}+\left[\mathbf{K}-\omega^{2}\left(\mathbf{M}-\mathbf{G}-\mathbf{K}_{\omega}\right)\right. \\
\left.+\mathrm{i} \omega \mathbf{C}_{\mathrm{n}}-\mathrm{i}\left(\omega-\omega_{\mathrm{d}}\right) \mathbf{C}_{\mathrm{d}}\right] \mathbf{r}=\omega^{2} \mathbf{F}_{\mathrm{r}}+\mathbf{F}_{\mathrm{n}} \mathrm{e}^{-\mathrm{i} \omega t}, \quad(30)
\end{gathered}
$$

where $\mathbf{r}=\mathbf{q e}^{-\mathrm{i} \omega t}$. Equation (30) is equivalent to Eq. (20).

Operating as in the previous case, the following expression for the control-gain matrix yielding an ideal proportional controller is obtained:

$$
\mathbf{P}_{i}=-c_{\mathrm{d}_{i}}\left[\begin{array}{cccc}
1 & 0 & 0 & \omega-\omega_{\mathrm{d}} \\
0 & 1 & -\left(\omega-\omega_{\mathrm{d}}\right) & 0
\end{array}\right] .
$$

Similarly, it is possible to synthesize nonrotating damping using a rotor-fixed active damper by introducing the following control-gain matrix:

$$
\mathbf{P}_{i}=-c_{\mathrm{d}_{i}}\left[\begin{array}{cccc}
1 & 0 & 0 & \omega \\
0 & 1 & -\omega & 0
\end{array}\right] .
$$

\section{CONCLUSIONS}

The role of nonsynchronous damping in rotordynamics, has been studied. In particular, its effect on the stability and the unbalance response of a Jeffcott rotor has been dealt with in detail. 
Co-rotating damping has been shown to decrease the stability of the system. By increasing the rotational speed of the damper, the stability of forward modes is reduced.

Moderate counter-rotating damping, i.e. a counter-rotating damping with low damping coefficient or characterized by a speed with low absolute value, increases the stability of forward modes while reducing that of backward modes, which however remain stable. A value of the product of the damping coefficient and the speed causing both forward and backward modes to be always stable was found.

Backward modes can become unstable, while forward ones show no threshold of instability, if a stronger counter-rotating damping is present.

A general model for an axi-symmetric rotating machine with many degrees of freedom was described. Even if no general result can be obtained in this case, the conclusions seen for the Jeffcott rotor still hold in a qualitative way.

Nonsynchronous rotating dampers require the presence of an element rotating at a speed which is different from that of the rotor and thus have limited practical applications. However, as active dampers can synthesize nonsynchronous damping without the need of a material object rotating at the required speed, there is no difficulty in incorporating this form of damping in systems running on magnetic bearings.

If active dampers are used, the devices synthesizing nonrotating damping or damping rotating at any arbitrary speed can all be located within the rotor. This is particularly interesting in statorless rotors as spinning spacecraft, in which the only conventional way of applying nonrotating damping is to introduce de-spun devices.

\section{LIST OF SYMBOLS}

$\begin{array}{ll}c & \text { damping coefficient } \\ k & \text { stiffness } \\ \mathrm{i} & \text { imaginary unit }(\mathrm{i}=\sqrt{-1}) \\ m & \text { mass }\end{array}$

\begin{tabular}{ll}
$\mathbf{q}$ & complex coordinates vector \\
$r$ & ratio $\omega_{\mathrm{d}} / \omega$ \\
$\mathbf{r}$ & complex coordinate vector, rotor-fixed \\
& frame \\
$t$ & time \\
$\mathbf{u}$ & input vector \\
$v$ & velocity \\
$z$ & complex coordinate \\
$\mathbf{Z}$ & state vector \\
$x, y$ & real coordinates \\
$\mathbf{y}$ & output vector \\
$\mathbf{A}$ & dynamic matrix \\
$\mathbf{B}$ & input-gain matrix \\
$\mathbf{C}$ & damping matrix \\
$F$ & external force \\
$\mathbf{F}$ & external force vector \\
$\mathbf{G}$ & gyroscopic matrix \\
$\mathbf{K}$ & stiffness matrix \\
$\mathbf{K}$ & centrifugal stiffness matrix \\
$\mathbf{M}$ & mass matrix \\
$\mathbf{P}$ & constant-gain matrix \\
$\varepsilon$ & eccentricity \\
$\zeta$ & damping ratio \\
$\eta$ & loss factor \\
$\lambda$ & whirl speed \\
$\omega$ & spin speed \\
$\omega_{\mathrm{cr}}$ & critical speed of the undamped system \\
$\mathfrak{R}()$ & real part \\
$\Im()$ & imaginary part \\
& \\
\hline &
\end{tabular}

\section{Superscript}

nondimensional

\section{Subscripts}

c control

d nonsynchronous

n nonrotating

$\mathrm{r} \quad$ rotating

\section{References}

Crandall, S.H. (1956) Engineering Analysis: A Survey of Numerical Procedures, Mc-Graw Hill, New York.

Crandall, S.H. (1982) A heuristic explanation of journal bearing instability, in Rotordynamic Instability Problems in Turbomachinery, NASA CP-2250. 
Crandall, S.H. (1995) Rotordynamics, in Nonlinear Dynamics and Stochastic Mechanics, W. Kliemann and N.S. Namachchivaya (Eds.), CRC Press, Boca Raton, pp. 1-44.

Dimentberg, F.M. (1961) Flexural Vibrations of Rotating Shafts, Butterworths, London.

Genta, G. (1998) Vibration of Structures and Machines, 3rd edn., Springer, New York.

Genta, G., Brusa, E. and Molina, E. (1996) A simple demonstrator for rotordynamics, Proc. Int. Conf. on Material Engineering - XXV Convegno Nazionale AIAS, Gallipoli, Italy, pp. 631-638.

Genta, G. and Brusa, E. (1997) Active stabilization of a fastspinning multibody spacecraft, 48th IAF - Int. Astronautical Congress.

Lalanne, M. and Ferraris, G. (1990) Rotordynamics Predictions in Engineering, Wiley, New York.

Lund, J.W. (1987) Stability and damped critical speeds of a flexible rotor in fluid-film bearings, in Rotating Machinery Dynamics, ASME, Vol. 1.

Muszynska, A. (1986) Whirl and whip-rotor/bearing stability problems, Journal of Sound and Vibration, 110(3), 443-462.

Muszynska, A. (1989) Anti-swirl arrangements prevent rotor/ seal instability, Journal of Vibration, Acoustics, Stress and Reliability Design, 111, 156-162.
Muszynska, A. (1990) Multimode whirl and whip in rotor/ bearing systems, Proceedings of the II Int. Symp. on Transport Phenomena, Dynamics and Design of Rotating machinery, Part II.

Muszynska, A. (1991) One lateral mode isotropic rotor response to nonsynchronous excitation, BRDRC Report 4/1991, Bently Rotor Dynamics Research Corp.

Muszynska, A. (1995) Modal testing of rotors with fluid interaction, Int. Journal of Rotating Machinery, 1(2), 83-116. Muszynska, A. and Bently, D. (1989) Fluid-generated instabilities of rotors, Orbit, $\mathbf{1 0}(1)$.

Muszynska, A. and Grant, J.W. (1991) Stability and instability of a two-mode rotor supported by two fluid-lubricated bearings, Journal of Vibration and Acoustics, 113, 316-324.

Nobili, A.M., Bramanti, D., Genta, G. et al. (1998) Proposed noncryogenic, nondrag-free test of the equivalence principle in space, New Astronomy, 3, 175-218.

Ramanujam, G. and Bert, C.W. (1983) Whirling and stability of flywheel systems, Part II: Comparison of numerical results obtained with combined and lumped parameter models, Journal of Sound and Vibration, 88(3), $399-420$. 


\section{ait \\ ENERGY MATERIALS}

M A N E Y publishing

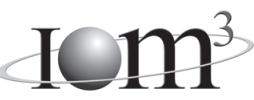

\section{Materials Science \& Engineering for Energy Systems}

Maney Publishing on behalf of the Institute of Materials, Minerals and Mining

The Institute of Materials, Minerals \& Mining

Economic and environmental factors are creating ever greater pressures for the efficient generation, transmission and use of energy. Materials developments are crucial to progress in all these areas: to innovation in design; to extending lifetime and maintenance intervals; and to successful operation in more demanding environments. Drawing together the broad community with interests in these areas, Energy Materials addresses materials needs in future energy generation, transmission, utilisation, conservation and storage. The journal covers thermal generation and gas turbines; renewable power (wind, wave, tidal, hydro, solar and geothermal); fuel cells (low and high temperature); materials issues relevant to biomass and biotechnology; nuclear power generation (fission and fusion); hydrogen generation and storage in the context of the 'hydrogen economy'; and the transmission and storage of the energy produced.

As well as publishing high-quality peer-reviewed research, Energy Materials promotes discussion of issues common to all sectors, through commissioned reviews and commentaries. The journal includes coverage of energy economics and policy, and broader social issues, since the political and legislative context influence research and investment decisions.

\section{CALL FOR PAPERS}

Contributions to the journal should be submitted online at http://ema.edmgr.com

To view the Notes for Contributors please visit: www.maney.co.uk/journals/notes/ema

Upon publication in 2006, this journal will be available via the Ingenta Connect journals service. To view free sample content online visit: www.ingentaconnect.com/content/maney

For further information please contact:

Maney Publishing UK

Tel: +44 (0)113 2497481 Fax: +44 (0)1132486983 Email: subscriptions@maney.co.uk

or

Maney Publishing North America

Tel (toll free): 8662975154 Fax: 6173546875 Email: maney@maneyusa.com

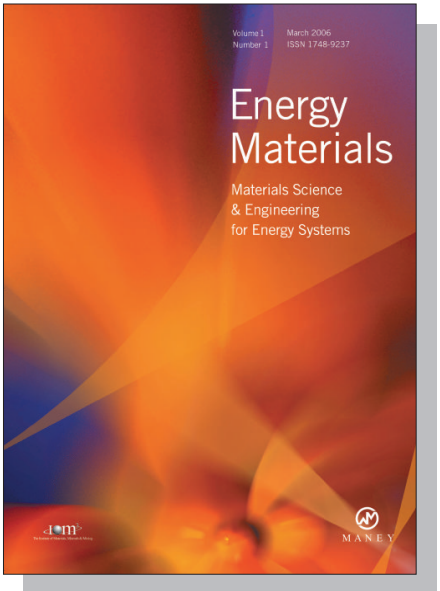

EDITORS

Dr Fujio Abe

NIMS, Japan

Dr John Hald, IPL-MPT, Technical University of Denmark, Denmark

Dr R Viswanathan, EPRI, USA

\section{SUBSCRIPTION INFORMATION}

Volume 1 (2006), 4 issues per year

Print ISSN: 1748-9237 Online ISSN: 1748-9245

Individual rate: $£ 76.00 / U S \$ 141.00$

Institutional rate: $£ 235.00 /$ US $\$ 435.00$

Online-only institutional rate: $£ 199.00 / U S \$ 367.00$

For special $\mathrm{IOM}^{3}$ member rates please email

subscriptions@maney.co.uk

\section{For further information or to subscribe online please visit www.maney.co.uk}



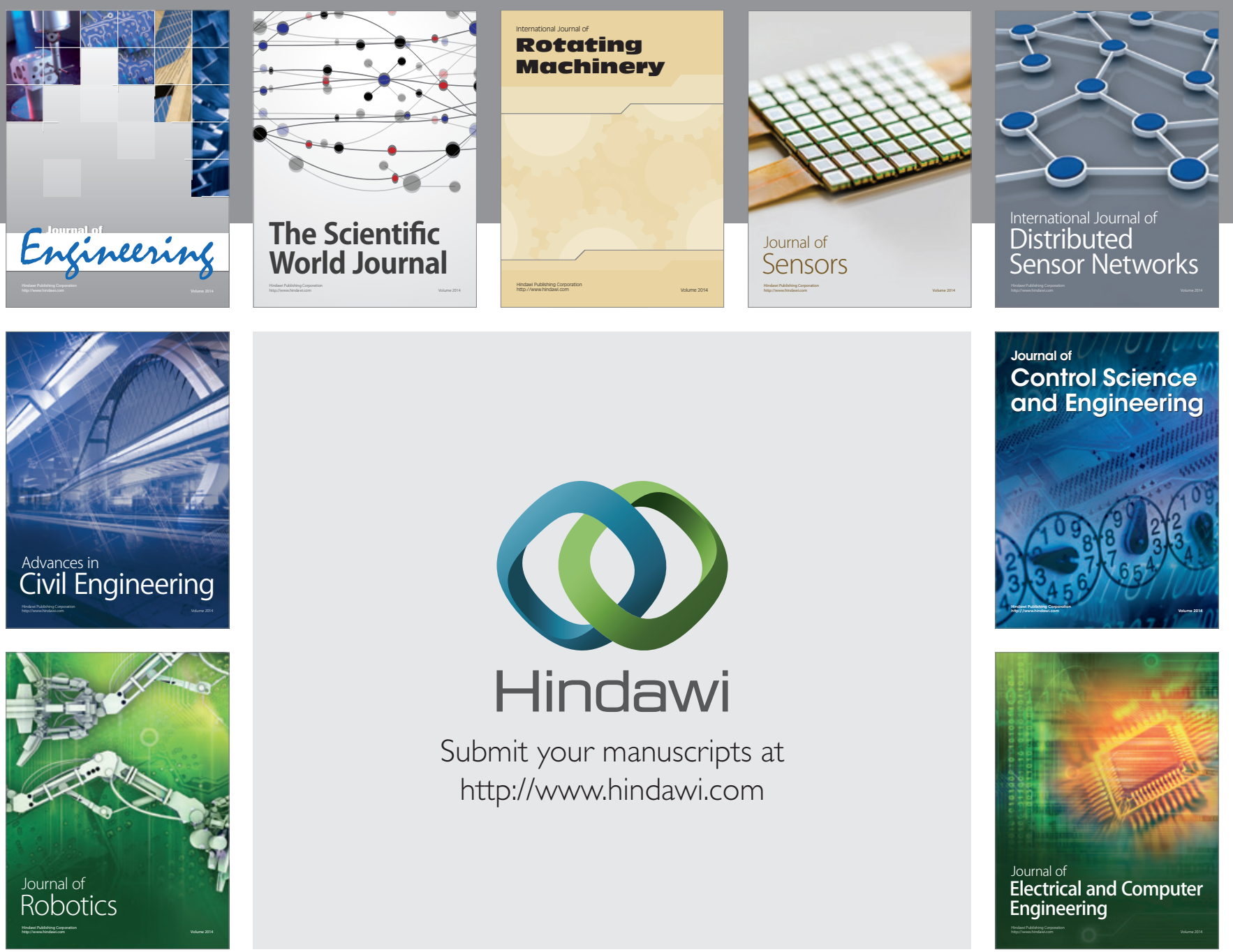

Submit your manuscripts at

http://www.hindawi.com
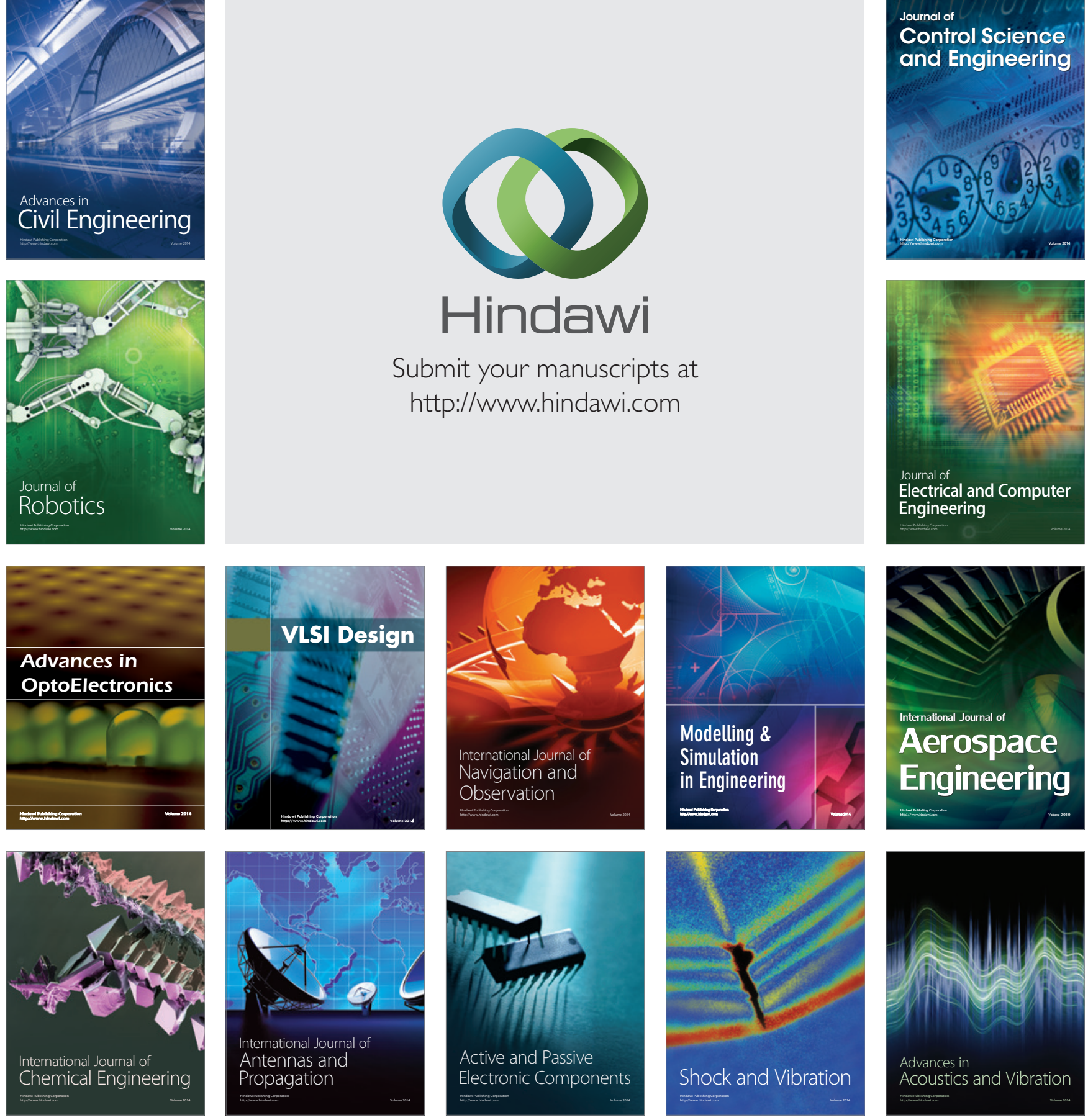